\title{
Efficient Face Detection Method using Modified Hausdorff Distance Method with C4.5 Classifier and Canny Edge Detection
}

\author{
Neelima Singh \\ Research Scholar \\ Computer Science and \\ Engineering Department \\ Samrat Ashok Technological \\ Institute, Vidisha, M. P.
}

\author{
Satish Pawar \\ Assistant Professor \\ Computer Science and \\ Engineering Department \\ Samrat Ashok Technological \\ Institute, Vidisha, M. P.
}

\author{
Yogendra Kumar Jain \\ Head of Department \\ Computer Science and \\ Engineering Department \\ Samrat Ashok Technological \\ Institute, Vidisha, M. P
}

\begin{abstract}
With the rapid growth of population and technology, security problems become a major issue due to abnormal human behaviors. Recently researches have been motivated towards automatic human face detection from still image or from moving image. Present human face detection system leads computation inaccuracies i.e. higher degree of false negative rate. In this paper, a multilevel hybrid model has been proposed for face detection. In the proposed work, we initially use C4.5 classifier so that foreground and background images can be differentiated, as a result of which search space can be reduced. After that skin color model has been applied to detect the skin region which is followed by canny edge detection to detect the edges of skin region. In the last step, we use the Modified Hausdorff Distance Method which matches the pixel values and detects the faces with lower false negative rate.
\end{abstract}

\section{Keywords}

C4.5 Classifier, Modified Hausdorff Distance, $\mathrm{YCbCr}$ Color space model

\section{INTRODUCTION}

Face detection in images is a very emerging field among the most popular topics of research in the field of computer vision. With the function of localizing and extracting the face area from the background, face detection is an essential step towards many applications such as intelligent human computer interaction, face recognition, human face animation, video conferencing, content based image retrieval and surveillances. In real life image, human faces have to suffer from high degree of changeability in appearance due to different illumination intensity, occlusion, head rotation and object distance that leads to major challenges in face detection. The major part of the face detection systems assumes a definite orientation of the faces, for instance frontal or near frontal face to make things easier [1-3].

Face detection method has been classified into two: (i) Modelbased approach and (ii) Feature-based approach. According to first approach, a face is assumed to be a single unit. Many techniques have been used to symbolize faces such as Skin Color Regressive Model [4], Neural Network [5] [6], Probabilistic Distribution [7], Principal Component Analysis [8], Local Feature Analysis [9] [10] and Support Vector Machines [11]. According to second approach, a face can be seen as an assemblage of different parts with geometrical features such as eyes, nose, and mouth [12]. Face detection can be achieved by verifying to a certain degree of confidence that features extracted from images represent a face. Both the approaches are applicable for different application area and might be lead to large research space i.e. insufficient face geometry features, missing color information, etc.

Skin color recognition is a powerful and fundamental initial procedure in the face detection process. Many researchers developed different skin color models to identify prospective skin areas and then identify the faces by analyzing each face candidate's shape and local geometric information such as eye and lip positions [12].

In the proposed method, we have applied the fusion of $\mathrm{C} 4.5$ classifier, $\mathrm{YCbCr}$ color model and Modified Hausdorff Distance method to increase the detection accuracy. Color model has been applied to detect the skin regions but fails to identify the some faces or some parts of faces in different illumination conditions. Thus to reduce that limitation and to verify the skin region as face, we have applied the Modified Hausdorff Distance Method which detects the faces on the basis of minimum distance calculated between the neighbor pixels. This increases the accuracy the proposed system in terms of True Positive Rate (TPR).

\section{RELATED WORK}

In any face detection processing, first step must be finding the location of face in the image. But doing that is quite difficult because of the variance present in the image in terms of color, pose, location, etc. There are many methods for face detection have been developed by choosing color as the basic feature because it can increase the detection rate. But only color model can't give promising results. Several researchers have also been carried out their work in the field of face detection using features based detection, neural network based detection, segmentation, etc. Kovac et.al used color compensation and color constancy methods to eliminate the effects of illumination [13]. Initially, the color pixels of skin had been found and after that skin elements are segmented into regions. Then on behalf of geometric properties of face, they discarded the inappropriate regions of an image, so that remaining region represents face. But this method was unable to produce good results when the illumination condition becomes very extreme or having the complex backgrounds. To overcome this problem, Lin applied neural network along with the $\mathrm{YCbCr}$ color space so that faces can be detected in complex environment also [14]. He made the scheme in two stages. In first stage, the author used color and triangle based segmentation to search potential face regions. In the second stage, face verification has been done by multilayer feed 
forward neural network. By using the segmentation, the author increases the detection rate and also increases the ability to handle different illumination condition. But this method was not efficient for rotation invariant face detection. Thus, a new skin color modeling process is applied to the face segmentation process by Mohamed et. al. to detect the human faces from complex background and also detects the faces which are rotation invariant [15]. After segmentation, image enhancement is done to improve the features of face candidates. After that face regions are subjected to the face object classifier which is based on a modified Hausdorff distance. It has increased the accuracy of the method but couldn't achieve sufficient success rate. At the same time, an automatic face detection method based on modeling of geometric structure of the face had been introduced by Paul et. al [16]. This method improves the rotation invariant face detection rate and limits the search space. Higher face detection accuracy and improved time complexity had been achieved by the fusion of PCA based geometric modeling and SCM (Skin Color Modeling) method. Both PCA and SCM provide filtering of image in terms of pixel values to get the face location in spite of illumination condition and face invariance. But complex geometric structure requires better understanding for important facial features and threshold values which can be done by using some other methods along with PCA and SCM. Ghazali et.al also proposed a method for face detection based on $\mathrm{YCgCr}$ color space to detect the face from the very wide variety of faces and also uncertain of face positions [17]. After building the Gaussian model in $\mathrm{Cg}-\mathrm{Cr}$ space, a calculation of correlation coefficient had been performed between the given template and the candidates. Their main aim was to achieve high detection rate and low false positive rate over a wide range of facial variations in different illumination conditions, color and positions, but low false detection rate couldn't be achieved in complex backgrounds.

We have used C4.5 classifier before the color model to reduce the search space and canny edge detection along with modified Hausdorff Distance method to detect the faces. $\mathrm{YCbCr}$ color model has been used because it is independent in the illumination condition. Hence, our proposed method eliminates the problems which occur in the existing methods.

\section{PROPOSED METHODOLOGY}

The proposed method has introduced hybrid model that is used to detect the human faces in group images. First phase is responsible to apply the $\mathrm{C} 4.5$ classifier to differentiate the foreground and background image pixel and expel the non skin region apart from the skin region. Classifier C4.5 separates out background and foreground of the image on the basis of luminous intensity of pixels. Basically the luminous intensity of pixels depends upon the light focus on that pixel. Intensity of light focus on foreground is always different from the background. Thus, luminous intensity of pixel leads good classification of foreground and background pixels. After that in second phase, by using skin color modeling (YCbCr color space), we identify the skin region. And finally, we will apply modified Hausdorff distance method along with the canny edge detection to verify that the detected skin region is face. Representing an image by its edges has the advantage of reducing the amount of data required to be stored while retaining most of the image information. Canny method can produce thin edge with the smooth continuous pixels. Sobel edge detection method cannot produce smooth and thin edge compared to canny method. Canny method finds the edges of the skin region and then modified Hausdorff distance method detects the faces in the images on the basis of pixel values.

\subsection{Classification}

Proposed methodology use $\mathrm{C} 4.5$ classifier which is a decision tree algorithm [18]. It is used to classify the foreground and background image pixels of input multi-face image. C4.5 classifier needs to convert pixel values to their ASCII values for decision tree classification. In C4.5 classifier, the pixel values are based on training and test of pixels. C4.5 constructs decision trees from a set of training pixel value by using the concept of information entropy. The training data is a set

$$
T=T_{1}, T_{2}, \ldots \ldots T_{N}
$$

of already classified samples having different luminous intensity value where

$$
T_{i}=A_{1}, A_{2}, \ldots . A_{N}
$$

which is a combination of vector spaces $\mathrm{A}_{1}, \mathrm{~A}_{2}, \ldots, \mathrm{A}_{\mathrm{N}}$ that represent attributes or features of the sample.

The training data is augmented with a vector $\mathrm{C}=\mathrm{C}_{1}, \mathrm{C}_{2}$, ........ $\mathrm{C}_{\mathrm{M}}$ where $\mathrm{C}=\mathrm{C}_{1}, \mathrm{C}_{2}, \ldots \ldots . . \mathrm{C}_{\mathrm{M}}$ represents the class belonging to each sample. At each node of the tree, C4.5 chooses one attribute of the data that most effectively splits its set of samples into subsets enriched in one class or the other. Its criterion is the normalized information gain (difference in entropy) that results from choosing an attribute for splitting the data. The attribute with the highest normalized information gain is chosen to make the decision.

By using the divide-and-conquer algorithm, C4.5 creates a primary tree $\mathrm{P}$ as if every cases in $\mathrm{P}$ belongs to the similar class or the tree itself becomes leaf labelled if $\mathrm{P}$ is small, with the most regular class in $\mathrm{P}$.

However, select a set which is based on only attribute with two or more outcomes. Make that set as the root of the tree with one branch and for every result of test, partition $\mathrm{P}$ into equivalent subsets $\mathrm{P}_{1}, \mathrm{P}_{2}, \ldots, \mathrm{P}_{\mathrm{n}}$ according to the result for every case, and apply the same process recursively to every subset. Generally many sets are selected in the last step. C4.5 uses two interesting norms to rank the possible sets: information gain that uses the attribute selection measure, minimize total entropy of the subset $\{\mathrm{Pi}\}$, and default gain ratio which parts the information gain by information provided by the test results. The information gain algorithm can be described as:

- Select the attribute having the highest information gain

- $\quad$ P contains Pi tuples of class Ri for $i=\{1, \ldots, n\}$

- Information measure or estimated information is necessary to classify any arbitrary tuple:

$\mathrm{I}\left(\mathrm{P}_{1}, \mathrm{P}_{2}, \ldots \ldots \ldots, \mathrm{P}_{\mathrm{n}}\right)=-\sum_{\mathrm{i}=1}^{\mathrm{n}} \frac{\mathrm{Pi}}{\mathrm{P}} \log _{2}\left(\frac{\mathrm{Pi}}{\mathrm{P}}\right)$

- Entropy of attribute A with the values $\{\mathrm{a} 1, \mathrm{a} 2, \ldots, \mathrm{av}\}$ :

$E(A)=\sum_{j=1}^{m} \frac{P_{1 j}+\ldots \ldots \ldots+P_{n j}}{P} I\left(P_{1 j}, \ldots \ldots ., P_{n j}\right)$

- Information gain ( means how much can be gained) by branching on the attribute $\mathrm{A}$ :

$\operatorname{Gain}(\mathrm{A})=\mathrm{I}\left(\mathrm{P}_{1}, \mathrm{P}, \ldots \ldots \ldots, \mathrm{P}_{\mathrm{n}}\right)-\mathrm{E}(\mathrm{A})$

\subsection{Color Model}

Different types of color spaces have been used in the segmentation of the skin [14] [17]. In some cases, the classification of color has been performed using only chromo pixel (the property that produces the sensation of color other than the luminance), because it had been expected that chasm 
of the skin may be more sensitive to variations in the lighting if the luminance of the pixel is rejected.

Proposed methodology employed $\mathrm{YCbCr}$ color space because it represents in terms of luminance color $(\mathrm{Y})$ and chrominance components $(\mathrm{Cb}$ and $\mathrm{Cr}$ ). With the $\mathrm{Cb}$ and $\mathrm{Cr}$ values, skin will provide good coverage of different races. A classified pixel will be dyed if the values $[\mathrm{Cr}, \mathrm{Cb}]$ falls within the threshold distribution of the colour of the skin and gives the face portion in the colour image. The skin portion of an image should satisfy as follows:

$$
140<=\mathrm{Cr}<=165 ; 140<=\mathrm{Cb}<=195
$$

\subsection{Edge Detection}

Edge detection plays a very important role in the field of boundary line detection of human face. The Canny edge detection algorithm is a most constructive edge detector method which is based on such a criteria that leads by marking the edges as closely as possible to the actual edges to maximize localization, marking edges only once when a single edge exists for minimal response and minimizing the error rate [19]. Proposed methodology employed canny edge detection method for finding boundary edges. According to Canny edge detection, optimal filter satisfying the criteria may be effectively estimated by using the Gaussian function's first derivative.

Gaussian function $=G f(p, q)=\frac{1}{2 \pi \sigma^{2}} e^{-\frac{p^{2}+q^{2}}{2 \sigma^{2}}}$

$$
\begin{aligned}
& \text { First derivation of Gaussian function (wrt } \mathrm{p})= \\
& \frac{\partial \mathrm{Gf}(\mathrm{p}, \mathrm{q})}{\partial \mathrm{p}} \mathrm{axe} \\
& -\frac{\mathrm{p}^{2}+\mathrm{q}^{2}}{2 \sigma^{2}}
\end{aligned}
$$

In the first stage, smoothing of image has been done by convolving with a Gaussian filter. After this, the gradient of the image must be found by feeding the smoothed image through a convolution operation with the derivative of the Gaussian in both horizontal and vertical directions. The 2-D convolution operation can be described as

$I^{\prime}(p, q)=g(k, l) \otimes I(p, q)$

$I^{\prime}(p, q)=\sum_{k=-N}^{N} \sum_{l=-N}^{N} g(k, l) I(p-k, q-l)$

Where: $\mathrm{g}(\mathrm{k}, \mathrm{l})=$ convolution kernel

$\mathrm{I}(\mathrm{p}, \mathrm{q})=$ original image

$I^{\prime}(p, q)=$ filtered image

\subsection{Modified Hausdorff Distance Method}

Hausdorff Distance is the measure of similarity between 2 sets of points belonging to the same metric space [20]. The main advantage of this measure is that any type of connection between points is not required. This requirement overcomes through the implicit nearest neighbor correspondence between the points of sets. Since Hausdorff distance measures the closeness rather than exact superposition, thus it become more tolerant towards the disturbance in the locations of points.
Given the sets

$\mathrm{S}=\left\{\mathrm{S}_{1}, \mathrm{~S}_{2}, \ldots ., \mathrm{S}_{\mathrm{N}}\right\}$ and $\mathrm{T}=\left\{\mathrm{T}_{1}, \mathrm{~T}_{2}, \ldots ., \mathrm{T}_{\mathrm{M}}\right\}$,

Where $S_{i}$ and $T_{j}$ denotes pixels of two sets for

$\mathrm{S}_{\mathrm{i}}=1, \ldots \ldots \ldots \ldots, \mathrm{f}$ and $\mathrm{T}_{\mathrm{i}}=1, \ldots \ldots \ldots \ldots, \mathrm{g}$

The Hausdorff Distance is defined as

$\mathrm{H}(\mathrm{S}, \mathrm{T})=\max (\mathrm{h}(\mathrm{S}, \mathrm{T}), \mathrm{h}(\mathrm{T}, \mathrm{S}))$

where

$h(S, T)=\max _{f \in S} \min _{g \in T}\|f-g\|$

In this formula, $\|\mathrm{f}-\mathrm{g}\|$ is the difference over the elements of the two sets and obviously the contour pixels ( $f, g$ ) run over the sets of indexes $i=1, \ldots \ldots, f$ and $j=1, \ldots \ldots, g$.

In our case, this difference is taken to be the Euclidean distance between the two points. Since the original definition of the Hausdorff distance is rather sensitive to noise, we opted to use a more robust version of this metric that is the Modified Hausdorff distance [20] [21],

$h(S, T)=\frac{1}{f} \sum_{f \in S} \min _{g \in T}|| f-g \|$

$h(T, S)=\frac{1}{g} \sum_{g \in T} \min _{f \in S}\|f-g\|$

where $f$ and $g$ are the numbers of points in sets $\mathrm{S}$ and $\mathrm{T}$, respectively. By taking the average of the single point distances, it decreases the impact of outliers which makes it more suitable for pattern recognition purposes.

In proposed methodology, selection of edge of facial feature of human skin object has been created by using the canny edge detector which will be further evaluated by using modified Hausdorff distance method. Modified Hausdorff distance is used as the base for the object matching purpose. Thresholding is needed to decide the adequate threshold value which acts as a cut-off point between face and non face. Using a series of pixel value at the boundary level, the edges are obtained after applying canny edge, on the basis of which mean Hausdorff distance will be generated.

\section{EXPERIMENTAL RESULT AND ANALYSIS}

The proposed method has been performed over 100 images of the Groups Dataset [23] and provides the promising result. The EFDMDCC (Efficient Face Detection Method Using Modified Hausdorff Distance Method with C4.5 Classifier and Canny Edge Detection) can detect large number of faces in the image in comparison with the FDMHD (Face Detection Based on Skin Color Modeling and Modified Hausdorff Distance). Here, our main goal is to increase the True Positive Rate (TPR) i.e., our method have to detect more number of faces in the images and therefore False Negative Rate (FNR) will be decreased. 


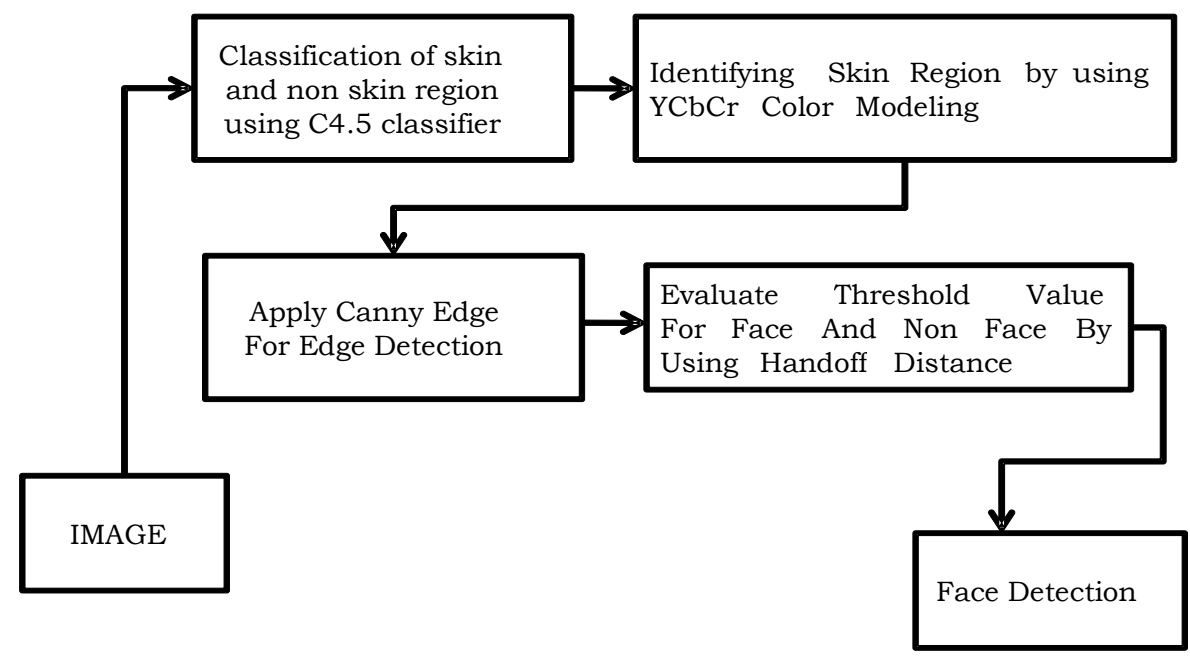

Fig 1: Proposed Architecture

For evacuating the performance of proposed multilevel hybrid model, TPR (True Positive Rate) and FNR (False Negative rate) have been used. Here, our main aim is to reduce the FNR.

True Positive Rate measures the proportion of actual positives which are correctly identified i.e., correctly identified faces in image to the total no. of faces in the image

$$
\mathrm{TPR}=\frac{\mathrm{TP}}{\mathrm{TP}+\mathrm{FN}}
$$

Similarly TNR (True negative rate) measures the proportion of actual negatives that are correctly rejected i.e.,

$$
\mathrm{TNR}=\frac{\mathrm{TN}}{\mathrm{FP}+\mathrm{TN}}
$$

FPR (False Positive rate) measures the proportion of actual negatives which has been incorrectly identified i.e.,

$$
\mathrm{FPR}=\frac{\mathrm{FP}}{\mathrm{FP}+\mathrm{TN}}
$$

Similarly FNR (False negative rate) measures the proportion of actual positives that are incorrectly rejected i.e.,

$$
\mathrm{FNR}=\frac{\mathrm{FN}}{\mathrm{TP}+\mathrm{FN}}
$$

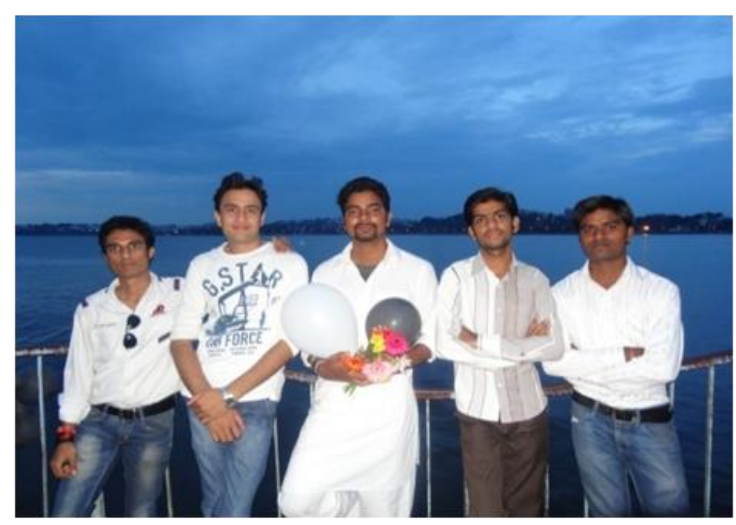

a. Original Image

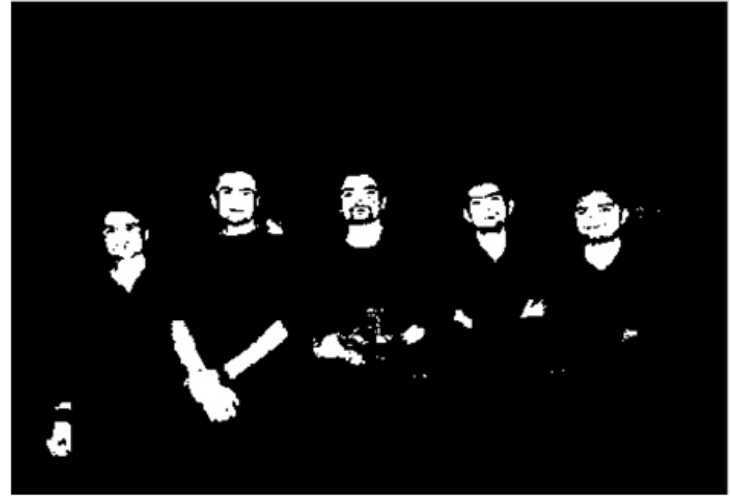

b. Image obtained after applying Classifier $\mathbf{C 4 . 5}$

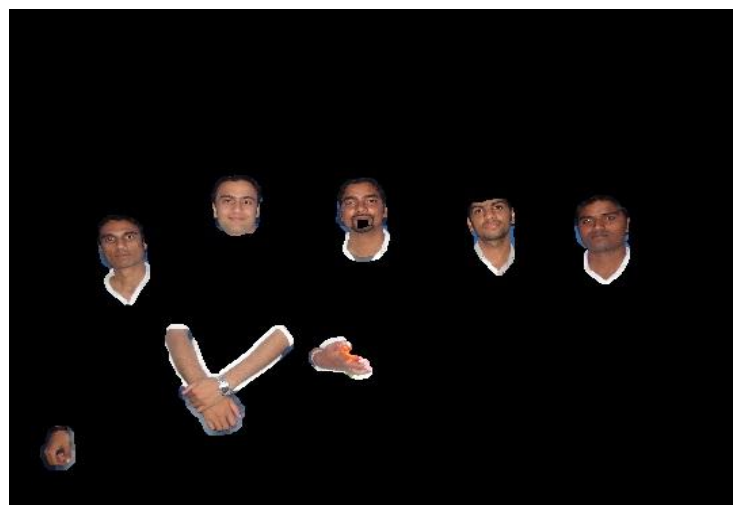

c. Skin region obtained after using $\mathrm{YCbCr}$ color space

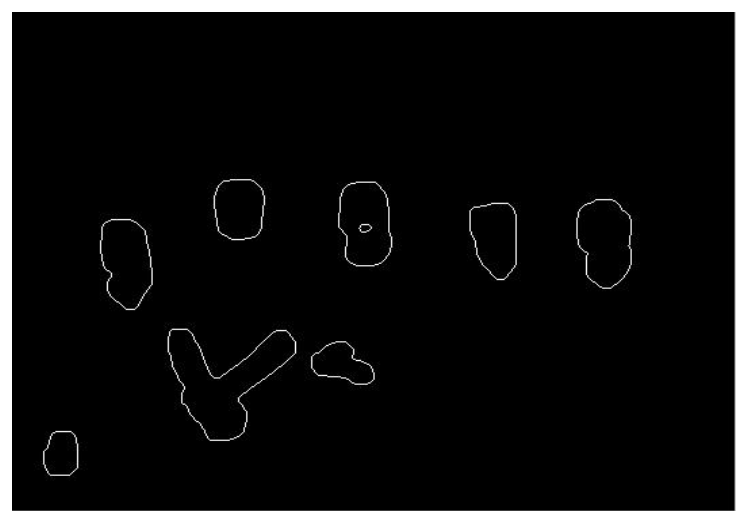

d. Edges detected by Canny Edge Detection 


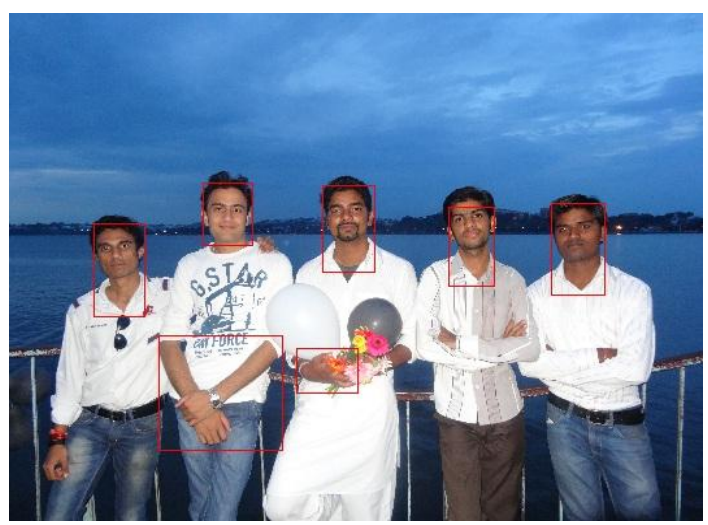

e. All faces are detected in the image by Modified Hausdorff Method

Fig 2: Step by Step Face detection Result using EFDHCC

In the proposed work, initially classifier $\mathrm{C} 4.5$ has been applied on the original image as shown in fig. 2(a) so that background can be eliminated from the image. A tree has

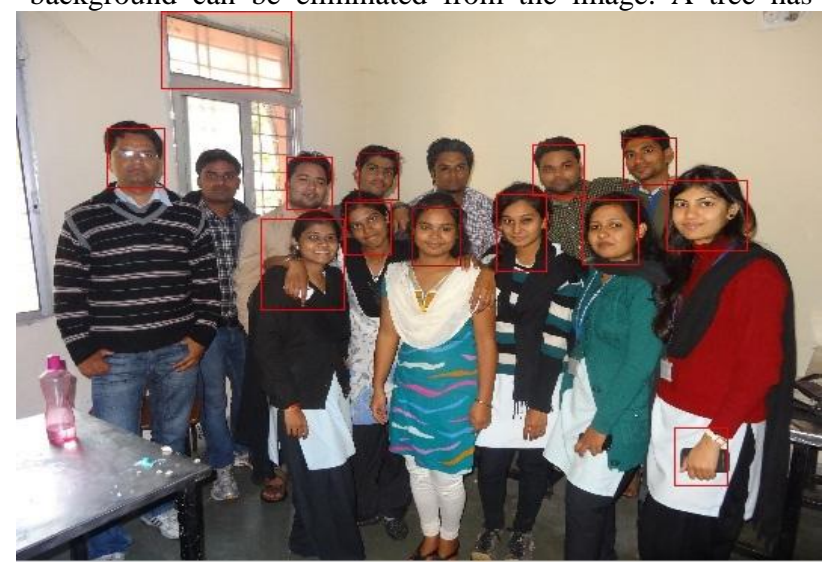

(a)

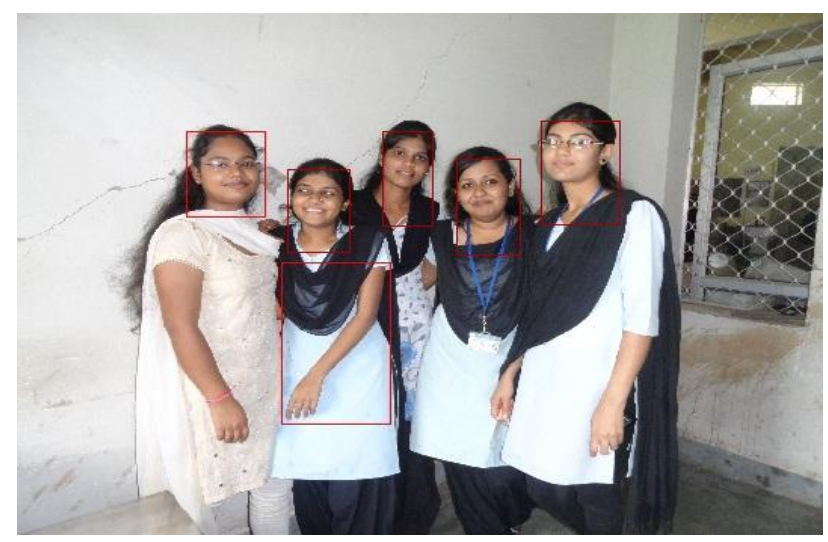

(c) been created on the basis of the value of color intensity of pixels and result of which background and foreground are distinguished as shown in fig. 2(b). After that, $\mathrm{YCbCr}$ color space model has been applied to identify only the skin region as shown in fig. 2(c). Color model is applied to the foreground obtained in the fig. 2(b). Finally, canny edge detection is applied along with Hausdorff Distance Method. After applying color model, canny edge detection is used to detect the edges as shown in fig. 2(d). After that Hausdorff Distance method has been applied on the image by using the edge as the threshold for calculating the distance between the pixel values to verify the skin region as the face. Pixels around edge having the minimum distance are considered as the face as shown in the fig. 2(e). Hausdorff Distance Method is used to verify the skin regions as face that has been detected by the color model. In the existing method, modified hausdorff had been used along with sobel operator which is not so efficient than canny operator.

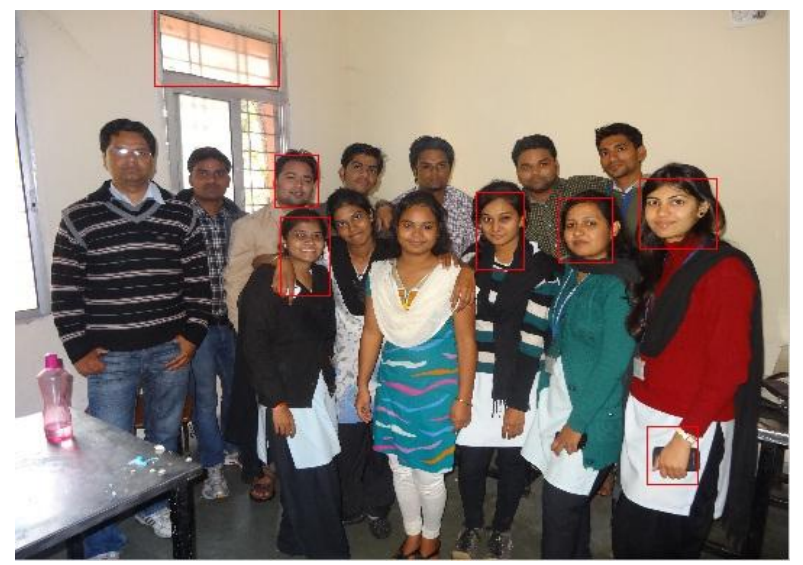

(b)

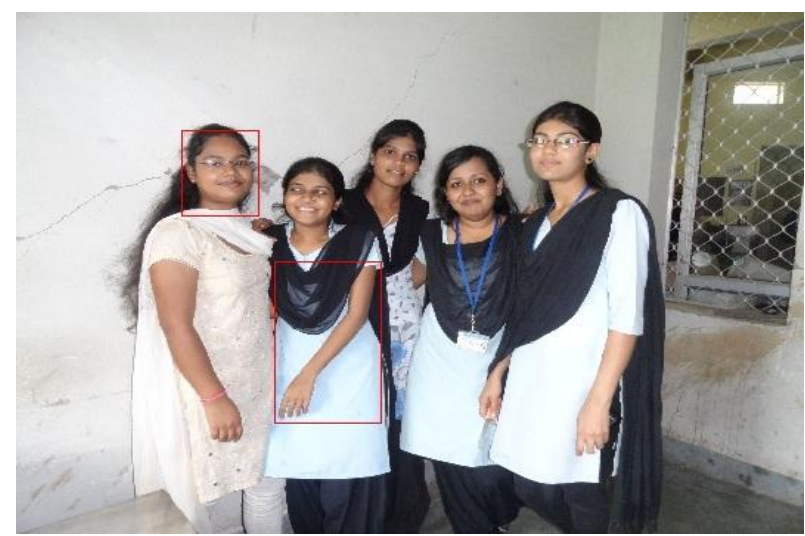

(d) 


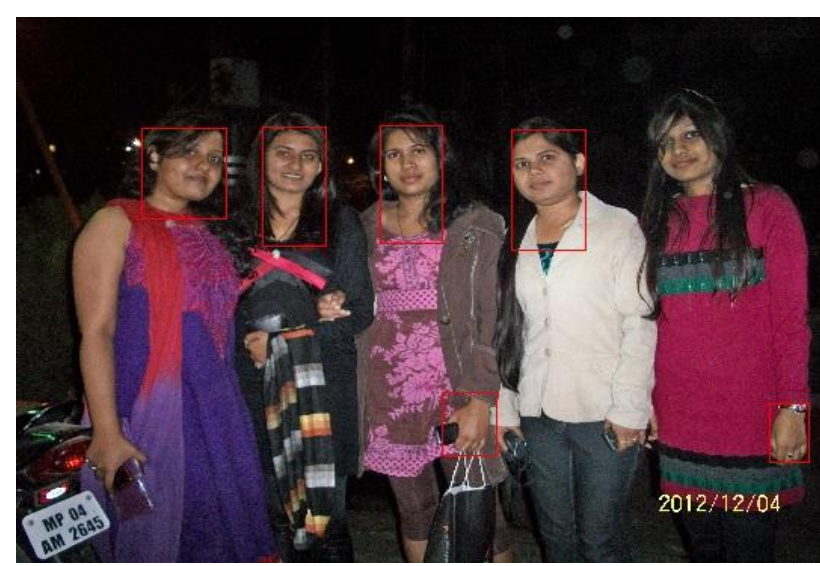

(e)

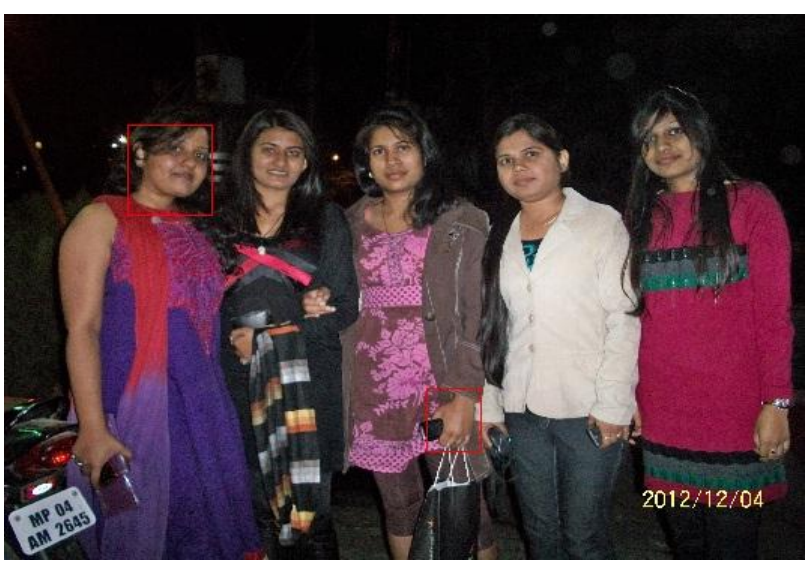

(f)

Fig. 3: Showing the comparison between the two methods- (a) (c) and (e) shows final face detection using EFDHCC and (b) (d) \& (f) shows final face detection using FDMHD

The result of other images also have been shown in fig. 3 to show the comparison between the two methods. After applying the face detection system in the images of database, it is also applied on the images other than the database to prove the effectiveness of the proposed method. Fig. 3, (a) (c) and (e) shows the result of proposed method over images other than the face database. 11 faces have been detected in fig. 3(a) out of 13 faces, all faces have been detected in the fig. 3 (c) and 4 faces have been detected out of 5 faces in the fig. 3(e). The result shows that the proposed method works effectively on other images also.

From the table 1, it has been shown that the average TPR of proposed work is $90.49 \%$ which is better than FDMHD i.e. $66.74 \%$ whereas FNR of proposed work is $8.71 \%$ which is less than FDMHD i.e. $33.23 \%$. This clearly shows that proposed method performs better than existing one.

Table 1: Table Showing Comparison between Existing and Proposed method

\begin{tabular}{|c|c|c|c|c|}
\hline & \multicolumn{2}{|c|}{ TPR } & \multicolumn{2}{c|}{ FNR } \\
\hline $\begin{array}{c}\text { Image } \\
\text { No. }\end{array}$ & FDMHD & EFDHCC & FDMHD & EFDHCC \\
\hline 1.jpg & 22.22 & 55.56 & 77.77778 & 44.4444 \\
\hline 2.jpg & 83.33 & 100.00 & 16.66667 & 0 \\
\hline 3.jpg & 66.67 & 66.67 & 33.33333 & 16.66667 \\
\hline 4.jpg & 57.14 & 85.71 & 42.85714 & 14.28571 \\
\hline 5.jpg & 85.71 & 85.71 & 14.28571 & 14.28571 \\
\hline 6.jpg & 71.43 & 85.71 & 28.57143 & 14.28571 \\
\hline 7.jpg & 71.43 & 85.71 & 28.57143 & 14.28571 \\
\hline 8.jpg & 83.33 & 100.00 & 16.66667 & 0 \\
\hline 9.jpg & 57.14 & 100.00 & 42.85714 & 0 \\
\hline 10.jpg & 66.67 & 83.33 & 33.33333 & 16.6667 \\
\hline 11.jpg & 83.33 & 100.00 & 16.66667 & 0 \\
\hline 12.jpg & 75.00 & 100.00 & 25 & 0 \\
\hline 13.jpg & 66.67 & 77.78 & 33.33333 & 22.22222 \\
\hline & & & & \\
\hline
\end{tabular}

\begin{tabular}{|c|c|c|c|c|}
\hline 14.jpg & 100.00 & 100.00 & 0 & 0 \\
\hline $15 . j p g$ & 100.00 & 100.00 & 0 & 0 \\
\hline $16 . j p g$ & 60.00 & 100.00 & 40 & 0 \\
\hline $17 . j p g$ & 80.00 & 100.00 & 20 & 0 \\
\hline $18 . j p g$ & 100.00 & 100.00 & 0 & 0 \\
\hline $19 . j p g$ & 60.00 & 100.00 & 40.00 & 0 \\
\hline 20.jpg & 20.00 & 100.00 & 80.00 & 0 \\
\hline $21 . j p g$ & 20.00 & 80.00 & 80.00 & 20.00 \\
\hline $22 . j p g$ & 38.46 & 84.61 & 61.53 & 15.38 \\
\hline
\end{tabular}

\section{CONCLUSION}

This paper presents a hybrid model for face detection from multi face image. Using C4.5 classifier reduces the search space which is a very critical task. Fusion of $\mathrm{YCbCr}$ color modeling and canny edge detection method with modified Hausdorff method provides higher face detection accuracy and decreases false negative rate. Experimental result confirms that the proposed method improves the face detection rate and limits the search space, thus making it very fast and efficient for large image databases. In future, False Positive Rate (FPR) can also be decreased by using some novel methods.

\section{REFERENCES}

[1] Liu C., "A bayesian discriminating features method for face detection" IEEE Trans. Pattern Analysis and Machine Intelligence, vol. 25, no. (6), pp. 725-740, 2003.

[2] Kim H., Lee S., and Cho N., "Rotation-invariant face detection using angular projections" Electron. Lett. , vol. (40), no. 12, pp. 726- 727, 2004.

[3] Suzuki Y., and Shibata T., "Multiple-clue face detection algorithm using edge-based feature vectors" Proc. ICASSP, vol. (5) , pp. 737-740, 2004.

[4] Padma Polash Paul, Md. Maruf Monwar, Marina Gavrilova, and Patrick Wang, "Rotation Invariant Multiview Face Detection Using Skin Color Regressive Model and Support Vector Regression", International Journal of 
Pattern Recognition and Artificial Intelligence, Vol. 24, no. (8), pp. 1261-1280, 2010.

[5] Hongming Zhang, Debin Zhao, Wen Gao and Xilin Chen, "Combining Skin Color Model and Neural Network for Rotation Invariant Face Detection", Springer-Verlag Berlin Heidelberg, vol. 1948, pp. 237$244,2000$.

[6] Rowley, H.A., Baluja, S., Kanade, T. "Neural networkbased face detection", IEEE Transactions on Pattern Analysis and Machine Intelligence, vol. 20, no. (1), pp. 23 - 38, Jan. 1998

[7] Sung, K.-K., Poggio, T.,"Example-based learning for view-based human face detection", IEEE Transactions on Pattern Analysis and Machine Intelligence, vol. 20, no. (1), pp.39-51, Jan.1998.

[8] Hazem M. El-Bakry, "New fast principal component analysis for face detection", Journal of Advanced Computational Intelligence and Intelligent Informatics, vol. 11, no.(2), pp. 195-201, 2007

[9] Yasmin Andreu, Ramon A. Mollineda and Pedro GarciaSevilla, "Gender Recognition from a Partial View of the Face Using Local Feature Vectors", Pattern Recognition and Image Analysis Springer, vol. 5524, pp. 481-488, 2009.

[10] Seungmin Lee, Hogyun Lee, and Taekyong Nam "A Comparative Study of the Objectionable Video Classification Approaches Using Single and Group Frame Features", ICANN Springer, pp. 616-623, 2006.

[11] Yongjin Kwona, M. K. Jeongb, O.A. Omitaomub, "Adaptive support vector regression analysis of closedloop inspection accuracy", International Journal of Machine Tools \& Manufacture, Elsevier, vol. 46 no. (6), pp. 603-610, May 2006.

[12] Anima Majumder "Automatic and Robust Detection of Facial Features in Frontal Face Images" UKSim 13th International Conference on Modelling and Simulation , IEEE, pp. 331-336, April 2011.

[13] J. Kovac, P. Peer, F. Solina, "Illumination independent color-based face detection", $3^{\text {rd }}$ International Symposium on Image and Signal Processing and Analysis, IEEE, vol. 1, pp. 510-515, Sept 2003.

[14] Chiunhsiun Lin, "Face detection in complicated backgrounds and different illumination conditions by using $\mathrm{YCbCr}$ color space and neural network" Elsevier Transaction on Pattern Recognition Letters, vol. 28, no. (16), pp. 2190-2200, Dec 2007.

[15] Khalid Mohamed Alajel, Wei Xiang, and John Leis "Face Detection Based on Skin Color Modeling and Modified Hausdorff Distance" in 8th Annual IEEE Consumer Communications and Networking Conference, IEEE, pp.399-404, Jan 2011

[16] Padma Polash Paul , Marina Gavrilova "PCA Based Geometric Modeling for Automatic Face Detection" in International Conference on Computational Science and Its Applications , International Conference on ICCSA, IEEE, pp. 33-38, 2011

[17] Kamarul Hawari Bin Ghazali, Jie Ma, Rui Xiao, Solly Aryza lubis, "An Innovative Face Detection Based on YCgCr Color Space", Elsevier International Conference on Solid State Devices and Materials Science, vol. 25, pp. 2116-2124, April 2012.

[18] Ramirez, G. A. and Fuentes, Olac, "Face Detection Using Combinations of Classifiers", the2nd Canadian Conference on Computer and Robot Vision, IEEE, pp. 610-615, May 2005.

[19] Reema Ajmera and Namrata Saxena, "Face Detection in Digital Images Using Color Spaces and Edge Detection Techniques", IJARCSSE, vol. 3, no. (6), pp. 718-725, 2013.

[20] Albert Pujol and Juan Jose Villanueva, "A Supervised Modification Of The Hausdorff Distance For Visual Shape Classification", International Journal of Pattern Recognition and Artificial Intelligence, vol. 16, no. (3), pp. 349-359, 2002.

[21] Erdem Yoruk, Ender Konuko־Glu, Bulent Sankur And Jerome Darbon, "Shape-Based Hand Recognition", IEEE Transactions On Image Processing, vol. 15, no.(7), pp. 1803-1815, 2006

[22] M. P. Dubuisson And A. K. Jain, "A Modified Hausdorff Distance For Object Matching" 12th Int. Conf. Pattern Recognition, pp. 566-568, 1994.

[23] A. Gallagher, T. Chen, "Understanding Groups of Images of People", IEEE Conference on Computer Vision and Pattern Recognition, 2009. 